Research Paper

\title{
Plasma N-acetylputrescine, cadaverine and 1,3-diaminopropane: potential biomarkers of lung cancer used to evaluate the efficacy of anticancer drugs
}

\author{
Ran Liu ${ }^{1}$, Pei Li ${ }^{1}$, Cathy Wenchuan $\mathrm{Bi}^{2}$, Ran Ma ${ }^{3}$, Yidi Yin ${ }^{1}$, Kaishun $\mathbf{B i}^{1}$ and Qing $\mathbf{L i}^{1}$ \\ ${ }^{1}$ School of Pharmacy, Shenyang Pharmaceutical University, Shenyang, China \\ ${ }^{2}$ Division of Life Science and Center for Chinese Medicine, The Hong Kong University of Science and Technology, Hong Kong, \\ China \\ ${ }^{3}$ School of Basic Medical Sciences, Liaoning University of Traditional Chinese Medicine, Shenyang, China
}

Correspondence to: Qing Li, email: lqyxm@hotmail.com

Keywords: polyamine, SCCL, cancer and medication biomarkers, targeted metabolomics

Received: April 28, 2017

Accepted: June 16, 2017

Published: July 17, 2017

Copyright: Liu et al. This is an open-access article distributed under the terms of the Creative Commons Attribution License 3.0 (CC BY 3.0 ), which permits unrestricted use, distribution, and reproduction in any medium, provided the original author and source are credited.

\section{ABSTRACT}

Polyamines have been widely investigated as potential biomarkers for various types of cancers, including lung cancer, which is one of the most common causes of death from cancer worldwide. This study was carried out to evaluate the value of polyamines that serve as early diagnostic and cancer progression markers as well as drug evaluation for lung cancer (squamous cell carcinoma of lung, SCCL). SCCL was induced in Wistar rats by intratracheal instillation of 3-methylcholanthrene and treated with three different anti-cancer drugs, Aidi injections, fluorouracil, and a combination of them. After carcinogenesis for 28, 70 and 98 days and therapy for 28 and 56 days, the polyamine levels in plasma of $\mathrm{SCCL}$, healthy and treated rats were determined using a UHPLC-MS/MS assay base on the means of targeted metabolomics. Results showed that increased $\mathbf{N}$-acetylputrescine, cadaverine and 1,3-diaminopropane levels were associated with progression of SCCL. The levels of cadaverine and 1,3-diaminopropane returned to normal after administration of the three different kinds of anticancer drug. In addition, the suitability of using $\mathrm{N}$-acetylputrescine, cadaverine and 1,3-diaminopropane as biomarkers was confirmed by PLS-DA and ROC analysis. It can provide an innovative and effective way for the clinical diagnosis, prevention and treatment of lung cancer, and stimulate a theoretical basis for the design and development of new anticancer drugs. At the same time, this increased the clinical options for polyamines as cancer biomarkers.

\section{INTRODUCTION}

Lung cancer has been the most common form of cancer worldwide for several decades, and it is also the most common cause of death from cancer [1-3]. Detailed pathogenesis, effective early detection and the use of suitable drugs help in the effective therapy of lung cancer. Among these, the early recognition of lung cancer is crucial, especially in screening the high-risk populations, as more aggressive treatment may improve clinical outcomes. Also, accurate diagnosis is vital for the most suitable treatment of individual patients with lung cancer. Thus, there is an urgent need to identify sensitive and specific biomarkers for early diagnosis and the therapeutic targets for investigating the pathogenetic mechanism of lung cancer. At present, the two protein biomarkers, CYFRA21-1 and SCCAg, are the most widely used forthe early diagosis of lung cancer [4-6]. However, a single biosignature is not enough to distinguish between healthy and diseased individuals. Thus, a combination of biosignatures, such as antigens, carbohydrates, enzymes, steroids and small molecule cancer markers, could improve the chances of an early diagnosis [7-10].

Metabolomics is now an established methodology and has wide ranging applications in many fields. It aims to profile all low-molecular weight metabolites that are 
present in biological samples to increase understanding of the effect of a particular stimulus, from the disease or drug, which affects metabolic pathways [11]. Targeted and untargeted applications are used for the identification of etiological pathways and the discovery of biomarkers for the prediction, diagnosis, and prognosis of major chronic diseases [12]. Through targeted metabolomics, the identified pathways or biomarkers could be confirmed by quantification of a limited number of metabolites in samples which leads to improved limits of detection and quantification compared with the use of open profiling approaches to confirm the biomarkers [13-15].

Among the different identified pathways in living organisms, polyamines are essential for the cell growth and differentiation of normal and neoplastic tissues. Polyamines, including putrescine, cadaverine, spermidine, spermine, agmatine, $\mathrm{N}$-acetylputrescine, $\mathrm{N}$-acetylspermine, $\mathrm{N}$-acetylspermidine and 1, 3-diaminopropane, are metabolized by amino acid such as L-ornithine, lysine and L-arginine. An increasing number of reports in the literature have now identified polyamine catabolism is a contributory factor in the development of a variety of cancers [16-20]. Regarding research into the relationship between polyamine metabolic profiling and lung cancer, we recently published papers on the level of polyamine metabolome in human subjects who suffered from lung and liver cancer [21-23]. Our results demonstrated the significance of polyamine metabolism in lung cancer. Because of the difficulties from clinical sample collection, the role of early diagnosis and therapeutic evaluation of polyamines in lung cancer had not been carried out. However, it encouraged us to undertake this study to determine a possible interrelation between lung carcinoma cell proliferation and polyamine metabolism in the early stages of lung cancer. Furthermore, we also examined potential therapeutic applications by investigating potential pharmaceutical interventions in polyamine metabolism.

Currently, with advantages of multi-target and multi-coordinated system, Chinese medicine is being widely used in cancer treatment, and positive effects have been achieved. However, problems including incomplete functional mechanisms, have affected the application of compound traditional Chinese medicines (CTCM). So, it is important to carry out CTCM anticancer pharmacodynamic evaluation on the basis of a polyamine metabolism response spectrum, which will provide new information about the correlation between biological endogenous substances and CTCM pharmacodynamics.

In this report we described how we investigated the impact of lung cancer on polyamine metabolism by examining the plasma from 3-methylcholanthrene (MCA) induced SCCL rats. Then, we evaluated the altered polyamine metabolism in the presence of an anti-cancer medicine, a complex Chinese medicine, a commonly used chemotherapeutic treatment and a combination of them. By targeted metabolomics analysis, simultaneous determination of polyamine metabolic profiles to identify specific biomarkers for the early diagnosis of SCCL and sensitive biomarkers for anti-cancer monitoring had been developed innovatively as well as constructively. The results obtained could provide an effective way for the clinical diagnosis, prevention and treatment of lung cancer, and provide a theoretical basis for the design and development of new anticancer drugs. Furthermore, this increased the clinical options for polyamines as biomarkers of cancer.

\section{RESULTS}

\section{Histopathology study of SCCL rats and treated rats}

The rats with SCCL induced by MCA solution were similar to human SCCL [26, 27]. MCA solution was prepared in Iodinated oil Injection at a temperature of $70-80^{\circ} \mathrm{C}$ and $10 \%(\mathrm{~V} / \mathrm{V})$ diethylnitrosamine was added to promote cancer development. Then, $0.1 \mathrm{ml}$ MCA solution (containing $10 \mathrm{mg} \mathrm{MCA}$ and $0.01 \mathrm{ml} \mathrm{DEN}$ ) was instilled once intratracheally into the left inferior pulmonary lobe of the rats.

General observation and histopathologic examinations were used for the evaluation of the SCCL model. A significant tumor was found on the left inferior pulmonary lobe of the SCCL rats, identified by general observation, but no primary or metastatic tumor was found on the other organs while there were no differences in the incidence of any lesions between the three treatment and normal groups. Histopathologic examination identified marked multifocal nests after carcinogenesis for 28 days (Figure 1A). With the process of carcinogenesis, the cancer cells increased in volume and number, with the development of cystic lesions (Figure 1B) and infiltration into the surrounding lung tissue (Figure 1C), which proved that the SCCL model had been successfully established.

As shown in Figure 2, the left inferior pulmonary lobe of the treated rats were also examined by obtaining pathological sections. On the 70th day, the MCA rats were given an Aidi injection for 28 days, and it could be seen that the cancer cells had been taken up by inflammatory cells as well as infiltrated many lymphocytes and multinuclear giant cells (Figure 2A1). After treatment for 56 days, alveolar cells were clearly seen and pulmonary cells tended to return to normal (Figure 2A2), which indicated the effect on self-recovery and immunity was increased by Aidi injection. When the MCA infected rat were treated with 5-fluorouracil for 28 days, necrotic cancerous cells and non-inflammatory cells were observed (Figure 2B1). While, after treatment for 56 days, the pulmonary cells tend to return normal (Figure 2B2). However, importantly, the rats given combinated treatments exhibited self-recovery and their immunity were enhanced. It seems that the secondary reaction of the 
chemotherapeutic, 5-fluorouracil, might be reduced by the anti-cancer Chinese medicine. So, normal alveolar cells were seen in the rats given combined treatment during their period of medication (Figure 2C).

\section{Levels of CYFRA21-1 and SCCAg in the plasma of SCCL and treated rats}

Many studies have reported that CYFRA211(cytokeratinfragment 21-1) and SCCAg (squamous cell carcinoma associated antigen) can be used as specific biomarkers for the early diagnosis of SCCL [4-6]. However, there are few reports about the application to the screening of the therapeutic effects of anti-cancer drugs. So, the changes in the expression of CYFRA21-1 and SCCAg were studied during the stage of SCCL onset, development and treatment as well as confirmation of the biomarker role of polyamines.
The levels of CYFRA21-1 and SCCAg in rat plasma were measured by enzyme linked immunosorbent assay (ELISA) and the results are shown in Table 1. Continuously increased levels were found in SCCL rats compared with normal rats $(P<0.01)$, even during the initial stage of SCCL. Reduced levels of CYFRA21-1 and SCCAg were found during anticancer drug administration. Comparison of rats given Aidi-injection, 5-fluorouracil and combined treatment showed that there was no significant difference in CYFRA21-1 and SCCAg in the early stage of therapy. However, as the therapy continued, the levels decreased in the rats given combination treatment. These results showed the feasibility of screening the anti-cancer drug effect when CYFRA21-1 and SCCAg were used as markers, while the Aidi injection and the combination of Aidi injection and 5-fluorouracil had an effect on the SCCL.

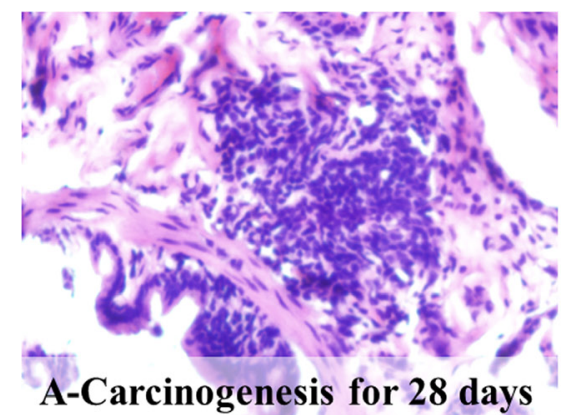

Figure 1: Histopathological photomicrographs of pathological lung sections obtained after intraperitoneal injection of the mixture of MCA and DEN solutions (haematoxylin and eosin (HE), ×40).
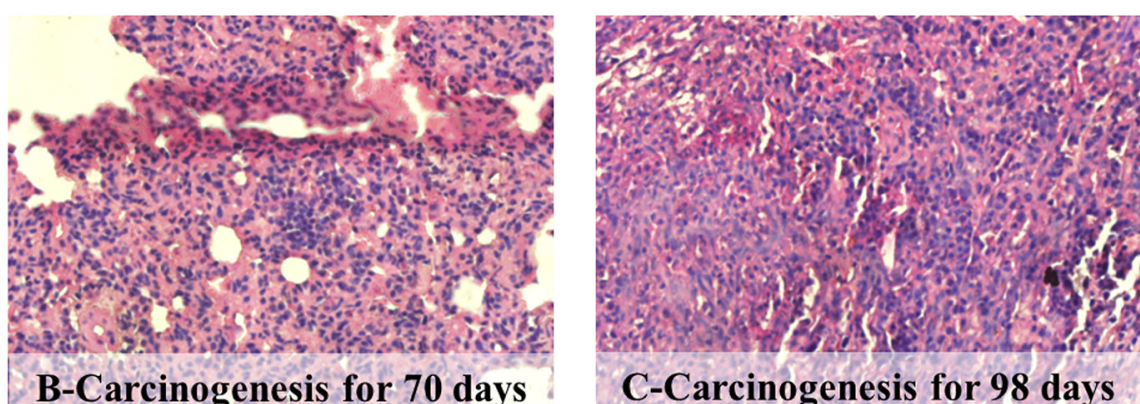

C-Carcinogenesis for 98 days
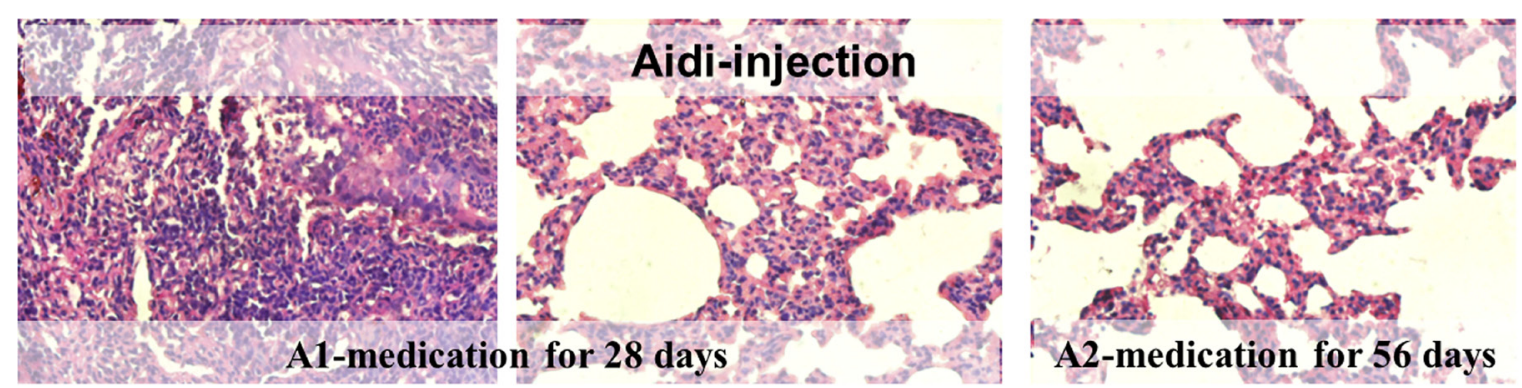
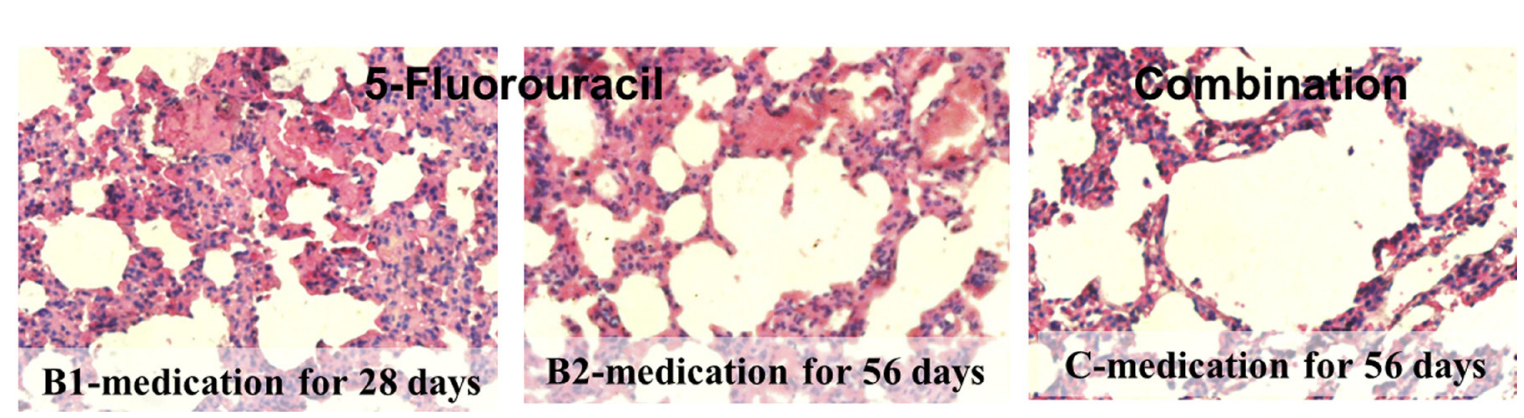

Figure 2: Histopathological photomicrographs of lung cancer rats after therapeutic treatment $(\mathrm{HE}, \times 40)$. (A) Aidiinjection; (B) 5-Fluorouracil; (C) Combination treatment. 
Table 1: Level of CYFRA21-1 and SCCAg in the different groups $(x \pm s) n=16$

\begin{tabular}{ccc}
\hline Group & CYFRA21 $(\mathbf{n g} / \mathbf{m l})$ & SCCAg $(\mathbf{n g} / \mathbf{m l})$ \\
\hline Normal Rats & $0.096 \pm 0.011$ & $0.865 \pm 0.092$ \\
SCCL Rats & $0.174 \pm 0.004^{*}$ & $0.180 \pm 0.062^{*}$ \\
Aidi-injection treated rats & $0.102 \pm 0.006$ & $0.937 \pm 0.096$ \\
5-fluorouracil treated rats & $0.097 \pm 0.009$ & $0.866 \pm 0.034$ \\
combination treated rats & $0.098 \pm 0.020$ & $0.900 \pm 0.031$ \\
\hline
\end{tabular}

${ }^{*} p<0.05$, compared with normal rats.

Table 2: Amounts of polyamine metabolomes in plasma $(\mathrm{ng} / \mathrm{mL})$ from Normal Rats $(n=8)$ and SCCL Rats $(n=8)$ from the 28th day to the 98th day during the experiment

\begin{tabular}{|c|c|c|c|c|c|c|}
\hline & \multicolumn{2}{|c|}{ 28th day } & \multicolumn{2}{|c|}{ 70th day } & \multicolumn{2}{|c|}{ 98th day } \\
\hline & Normal Rats & SCCL Rats & Normal Rats & SCCL Rats & Normal Rats & SCCL Rats \\
\hline 1,3-diaminopropane & $114.7 \pm 22.1$ & $135.1 \pm 9.7$ & $90.9 \pm 4.7$ & $928 \pm 458^{*}$ & $381.2 \pm 110.9$ & $1430 \pm 163^{*}$ \\
\hline putrescine & $3997 \pm 1083$ & $4345 \pm 1097$ & $3521 \pm 560$ & $5288 \pm 1812$ & $3708 \pm 1054$ & $3194 \pm 1751$ \\
\hline cadaverine & $383.7 \pm 106.0$ & $757.6 \pm 45.1^{*}$ & $258.3 \pm 119.5$ & $990 \pm 300^{*}$ & $1300 \pm 503$ & $4984 \pm 1371^{*}$ \\
\hline spermine & $6.623 \times 10^{4} \pm 0.534 \times 10^{4}$ & $4.830 \times 10^{4} \pm 1.816 \times 10^{4}$ & $2.204 \times 10^{4} \pm 0.475 \times 10^{4}$ & $1.950 \times 10^{4} \pm 1.627 \times 10^{4}$ & $1.497 \times 10^{4} \pm 0.708 \times 10^{4}$ & $1.590 \times 10^{4} \pm 0.564 \times 10^{4}$ \\
\hline spermidine & $1.391 \times 10^{4} \pm 0.118 \times 10^{4}$ & $9.78 \times 10^{3} \pm 1.94 \times 10^{4}$ & $7088 \pm 4494$ & $6373 \pm 4061$ & $6925 \pm 2124$ & $7804 \pm 2546$ \\
\hline $\mathrm{N}$-acetylputrescine & $2.51 \pm 1.23$ & $5.11 \pm 0.11^{*}$ & $3.82 \pm 0.58$ & $6.95 \pm 1.48^{*}$ & $3.06 \pm 1.02$ & $6.19 \pm 1.03 *$ \\
\hline $\mathrm{N}$-acetylspermine & $2.39 \pm 1.06$ & $2.01 \pm 0.29$ & $2.25 \pm 0.25$ & $3.34 \pm 2.13$ & $1.40 \pm 0.28$ & $1.31 \pm 0.32$ \\
\hline $\mathrm{N}$-acetylspermidine & $2.29 \pm 0.80$ & $1.22 \pm 0.33$ & $2.07 \pm 0.93$ & $2.04 \pm 1.29$ & $1.04 \pm 0.36$ & $1.93 \pm 0.84$ \\
\hline$\gamma$-aminobutyric acid & $1996 \pm 521$ & $2289 \pm 105$ & $2056 \pm 593$ & $4154 \pm 5452$ & $1725 \pm 761$ & $1415 \pm 673$ \\
\hline agmatine & $52.90 \pm 6.75$ & $58.33 \pm 3.84$ & $48.46 \pm 7.55$ & $75.93 \pm 45.61$ & $77.34 \pm 83.42$ & $43.90 \pm 12.91$ \\
\hline L-arginine & $2.001 \times 10^{4} \pm 0.279 \times 10^{4}$ & $1.468 \times 10^{4} \pm 0.244 \times 10^{4}$ & $1.928 \times 10^{4} \pm 0.263 \times 10^{4}$ & $1.623 \times 10^{4} \pm 1.032 \times 10^{4}$ & $1.659 \times 10^{4} \pm 0.535 \times 10^{4}$ & $1.750 \times 10^{4} \pm 0.225 \times 10^{4}$ \\
\hline lysine & $3.066 \times 10^{4} \pm 0.833 \times 10^{4}$ & $3.377 \times 10^{4} \pm 0.376 \times 10^{4}$ & $2.441 \times 10^{4} \pm 0.625 \times 10^{4}$ & $3.386 \times 10^{4} \pm 0.716 \times 10^{4}$ & $2.169 \times 10^{4} \pm 0.547 \times 10^{4}$ & $2.467 \times 10^{4} \pm 1.064 \times 10^{4}$ \\
\hline L-ornithine & $1.874 \times 10^{4} \pm 0.984 \times 10^{4}$ & $5.236 \times 10^{4} \pm 2.258 \times 10^{4}$ & $1.376 \times 10^{4} \pm 0.369 \times 10^{4}$ & $6.198 \times 10^{4} \pm 2.650 \times 10^{4 *}$ & $6.505 \times 10^{4} \pm 4.269 \times 10^{4}$ & $4.061 \times 10^{4} \pm 2.977 \times 10^{4}$ \\
\hline $\begin{array}{l}\text { S-adenosyl-L- } \\
\text { methionine }\end{array}$ & $357.6 \pm 19.7$ & $282.0 \pm 6.0^{*}$ & $160.8 \pm 11.3$ & $246.7 \pm 87.3$ & $116.2 \pm 45.8$ & $152.8 \pm 60.6$ \\
\hline
\end{tabular}

(mean $\pm \mathrm{SD}) .{ }^{*} p<0.05$, compared with normal rats.

\section{Polyamine metabolic profile in SCCL and treated rats}

The levels of 14 polyamine metabolomes in each plasma sample were determined from calibration curves (Supplementary Table 3 listed the detailed results of the method validation). Table 2 summarizes the concentrations (mean \pm SD) of the fourteen plasma polyamine metabolomes in SCCL rats and normal rats. On the 28th day after carcinogenesis, compared with normal rats, most of the polyamine levels in the rats with cancer were similar to those of the normal rats except for an increased level of putrescine and $\mathrm{N}$-acetylputrescine as well as reduced S-adenosylL-methionine $(p<0.05)$. This suggested that when the lung cell carcinoma appeared, the polyamine metabolism, especially the pathway related to putrescine, was increased which was why we found increased levels of putrescine in body fluids. Similarly, on the 70th day after carcinogenesis, the polyamine levels were significantly increased for cadaverine, $\mathrm{N}$-acetylputrescine, 1,3-diaminopropane and L-ornithine. It seems that the consumption of putrescine, especially the pathway from L-ornithine to
$\mathrm{N}$-acetylputrescine, was disturbed during the SCCL period. On the 98th day after carcinogenesis, compared with normal rats, the polyamine levels in the SCCL rats were increased for cadaverine, $\mathrm{N}$-acetylputrescine and 1,3-diaminopropane. It has been shown that the increased polyamine metabolism is a typical feature of lung caner malignancy, and the catabolism or transmission of $\mathrm{N}$-acetylputrescine seems to be interfered obviously. In summary, N-acetylputrescine can be used as a potential biomarker of SCCL.

As indicated in Table 3, the concentrations (mean $\pm \mathrm{SD}$ ) of the fourteen plasma polyamine metabolomes in treated rats differed significantly according to the treatments used. On the 70th day after carcinogenesis, when the rats has been given anti-cancer drugs for 28 days, the levels of cadaverine and 1,3-diaminopropane were significantly reduced in all the three drug treated rats, compared with SCCL rats. In addition, there were increased levels of $\mathrm{N}$-acetylputrescine and spermine in 5-fluorouracil treated rats, and a decreased level of lysine as well as an increased level of spermine in the rats given combination treatment. Then, after being given anticarcinogen treatment for about 56 days, slight 
Table 3: Amounts of polyamine metabolomes in plasma $(\mathrm{ng} / \mathrm{mL})$ from Aidi injection treated rats $(n=8)$, 5-fluorouracil treated rats $(n=8)$ and combination treated rats from the 70th to the 98th day during the experiment

\begin{tabular}{|c|c|c|c|c|c|c|}
\hline & \multicolumn{3}{|c|}{ 70th day } & \multicolumn{3}{|c|}{ 98th day } \\
\hline & Aidi & 5-fluorouracil & Combination & Aidi & 5-fluorouracil & Combination \\
\hline 1,3-diaminopropane & $682.2 \pm 407.5^{*}$ & $147.9 \pm 47.4^{*}$ & $250.3 \pm 97.4^{*}$ & $551.5 \pm 296.8^{*}$ & $798 \pm 138^{*}$ & $240.4 \pm 88.7^{*}$ \\
\hline putrescine & $4125 \pm 1616$ & $3893 \pm 395$ & $3379 \pm 1651$ & $4300 \pm 1263$ & $3729 \pm 953$ & $2613 \pm 1490$ \\
\hline cadaverine & $836 \pm 158^{*}$ & $665.2 \pm 281.9^{*}$ & $399.2 \pm 194.2 *$ & $839 \pm 424 *$ & $1791 \pm 353^{*}$ & $323.6 \pm 158.4^{*}$ \\
\hline spermine & $3.776 \times 10^{4} \pm 2.130 \times 10^{4}$ & $4.862 \times 10^{4} \pm 1.554 \times 10^{4 *}$ & $4.515 \times 10^{4} \pm 2.034 \times 10^{4 *}$ & $3.064 \times 10^{4} \pm 2.138 \times 10^{4}$ & $1.500 \times 10^{4} \pm 0.319 \times 10^{4}$ & $4.163 \times 10^{4} \pm 1.606 \times 10^{4}$ \\
\hline spermidine & $9.03 \times 10^{3} \pm 2.79 \times 10^{3}$ & $1.137 \times 10^{4} \pm 0.127 \times 10^{4}$ & $8.21 \times 10^{3} \pm 3.43 \times 10^{3}$ & $7719 \pm 1231$ & $9.25 \times 10^{3} \pm 0.60 \times 10^{3}$ & $7134 \pm 2148$ \\
\hline $\mathrm{N}$-acetylputrescine & $1.83 \pm 0.83^{*}$ & $2.45 \pm 0.74^{*}$ & $4.93 \pm 1.62$ & $1.68 \pm 1.84$ & $1.46 \pm 0.29 *$ & $1.33 \pm 0.65^{*}$ \\
\hline $\mathrm{N}$-acetylspermine & $1.60 \pm 0.73^{*}$ & $2.22 \pm 0.55$ & $1.94 \pm 0.60$ & $1.70 \pm 0.57$ & $1.65 \pm 0.29^{*}$ & $1.71 \pm 0.79$ \\
\hline $\mathrm{N}$-acetylspermidine & $1.57 \pm 0.45^{*}$ & $2.92 \pm 0.52$ & $2.08 \pm 0.59$ & $1.77 \pm 0.40$ & $2.07 \pm 1.98$ & $2.26 \pm 0.50$ \\
\hline$\gamma$-aminobutyric acid & $2282 \pm 508$ & $4219 \pm 1312$ & $2116 \pm 637$ & $2199 \pm 552$ & $1854 \pm 277$ & $1960 \pm 629$ \\
\hline agmatine & $46.23 \pm 19.67$ & $55.38 \pm 18.56$ & $46.09 \pm 11.08$ & $33.27 \pm 12.41$ & $41.41 \pm 10.68$ & $44.69 \pm 4.12$ \\
\hline L-arginine & $2.537 \times 10^{4} \pm 0.654 \times 10^{4}$ & $1.691 \times 10^{4} \pm 0.625 \times 10^{4}$ & $1.937 \times 10^{4} \pm 0.233 \times 10^{4}$ & $2.484 \times 10^{4} \pm 0.549 \times 10^{4}$ & $9.98 \times 10^{3} \pm 3.54 \times 10^{3}$ & $1.949 \times 10^{4} \pm 0.161 \times 10^{4}$ \\
\hline lysine & $2.967 \times 10^{4} \pm 0.899 \times 10^{4}$ & $4.833 \times 10^{4} \pm 2.406 \times 10^{4}$ & $2.263 \times 10^{4} \pm 0.645 \times 10^{4 *}$ & $2.708 \times 10^{4} \pm 0.804 \times 10^{4}$ & $2.078 \times 10^{4} \pm 0.439 \times 10^{4}$ & $2.132 \times 10^{4} \pm 0.392 \times 10^{4}$ \\
\hline L-ornithine & $6.298 \times 10^{4} \pm 4.663 \times 10^{4}$ & $5.968 \times 10^{4} \pm 0.817 \times 10^{4}$ & $1.047 \times 10^{5} \pm 0.926 \times 10^{5}$ & $7.406 \times 10^{4} \pm 5.766 \times 10^{4}$ & $1.028 \times 10^{5} \pm 0.492 \times 10^{5 *}$ & $1.226 \times 10^{5} \pm 0.549 \times 10^{5}$ \\
\hline $\begin{array}{l}\text { S-adenosyl-L- } \\
\text { methionine }\end{array}$ & $213.6 \pm 104.0$ & $443.8 \pm 192.5$ & $191.7 \pm 80.1$ & $217.7 \pm 156.3$ & $83.3 \pm 67.6$ & $156.3 \pm 55.1$ \\
\hline
\end{tabular}

(mean $\pm \mathrm{SD}) .{ }^{*} p<0.05$, compared with SCCL Rats.

alterations in plasma polyamine levels were observed in the rats given the three different reatments. Compared with SCCL rats, the levels of cadaverine and 1,3-diaminopropane were sequentially reduced in all the treated rats. Meanwhile, in the 5-fluorouracil treated rats, a reduced level of $\mathrm{N}$-acetylputrescine as well as increased levels of $\mathrm{N}$-acetylspermine and L-ornithine were observed. The changes in polyamine levels differed between the rats given Aidi injections and 5-fluorouracil injections. This might be because the different anti-cancer drugs had different mechanisms of action involving cancer therapy.

The results of the combination of polyamine metabolome and CYFRA21-1 and SCCAg determination showed both traditional Chinese medicine and synthetic drugs can alter the polyamine metabolic profiling and reduce the level of intracellular polyamines to inhibit proliferation in tumor cells. Also, the phenomenon of altered polyamine levels and polyamine metabolic pathways both confirmed the advantage of Aidi-injection, in which the compound traditional Chinese medicine composed of Cantharides, Ginseng, astragalus and Acanthopanax had the effect of suppressing tumor growth through inhibition of angiogenesis and regulating and strengtheningcellular immune function, with a multi-target and multi-coordinated effect on cancer treatment when combined with synthetic drugs.

In conclusion, both cadaverine and 1,3-diaminopropane have similar characteristics: an increased level in accordance with cancer progression and a gradual decrease according to the treatment until it becomes equal to that in normal rats. Thus, we concluded that cadaverine and 1,3-diaminopropane could serve as potential target biomarkers for monitoring medical treatments and predicting cancer remission.

\section{DISCUSSION}

\section{Identification of polyamine biomarkers}

For further screening of the markers of SCCL, the differences in polyamine metabolomes among healthy, SCCL and treated rats were evaluated using a supervised multivariate analysis. Partial least-squares discriminant analysis (PLS-DA) was performed using SIMCA 13.0. The corresponding variable importance in the projection (VIP values) were also calculated using the PLS-DA model.

PLS-DA was used to examine the targeted metabolic changes between the SCCL and healthy groups, and the SCCL and treated groups. As shown in Figure 3, the PLSDA score plot reveals a clear separation between healthy controls and SCCL subjects suffered carcinogenesis for 28 days, with good fitting and predictive performances $(\mathrm{R} 2 \mathrm{Y}=0.983, \mathrm{Q} 2=0.809)$, when using polyamines as indicators. Also, the loading scatter plot and coefficient plot shown in Figure 3 strongly support the effectiveness of using N-acetylputrescine and cadaverine to distinguish SCCL and healthy rats, since their M2.CoeffCS values were more marked than the others. With cancer development, the PLS-DA score plot could also distinguish between the healthy controls and SCCL subjects suffered carcinogenesis for 70 days, with $\mathrm{N}$-acetylputrescine, 
cadaverine and 1,3-diaminopropane as the most marked polyamines (as shown in Figure 4).

Interestingly, excellent separations were also achieved using PLS-DA analyses for rats given different treatments for 56 days versus SCCL rats (as shown in Figure 5). The results obtained showed that cadaverine and 1,3-diaminopropane can be used as indicators to effectively distinguish between treated and SCCL rats. This agrees with the results of the polyamine metabolic profiles in SCCL and treated rats using the T-test. Also, as SCCL treatment progressed, this difference became clearer.

Using the results obtained from the UHPLC-MS/MS and PLS-DA analysis, Receiver Operating Characteristic (ROC) curve analysis was further conducted by SPSS Statistics 18 software (SPSS Inc.) to evaluate the predictive power of each differential polyamine as we did with the target metabolomics on SCCL.

ROC curves were plotted on the basis of the set of optimal sensitivity and specificity values of a diagnostic test at different cutpoints. The cutpoint was determined for each potential biomarker by searching for those that yielded both high sensitivity and specificity. The area under the curve (AUC) was calculated by numerical integration of the ROC curves. The metabolite signature which had the largest area under the ROC curve was identified as having the strongest predictive power for detecting SCCL. The significance threshold of the area under the ROC curve was set at 0.85 . The detailed statistics of the area under the ROC curves (AUC), and the corresponding sensitivities and specificities for each of the potential biomarkers for SCCL prediction are listed in Figure 6.

From the results, N-acetylputrescine and 1,3-diaminopropane could be used as early biomarkers of SCCL, and cadaverine could be used for the development of biomarkers of SCCL. Moreover, cadaverine and 1,3-diaminopropane could be used as biomarkers for evaluating the therapeutic effect of anti-cancer drugs on SCCL.

Acetylation reactions may provide a way for cells to reduce the interactions of polyamines with different negatively charged compounds, such as DNA, and RNA, by reducing the number of positive charges they bear. Reduced excretion of $\mathrm{N}$-acetylputrescine may be one of the mechanisms by which the intracellular concentration of polyamines is uncontrolled, and this follows the development of SCCL. Also, 1,3-diaminopropane, derived
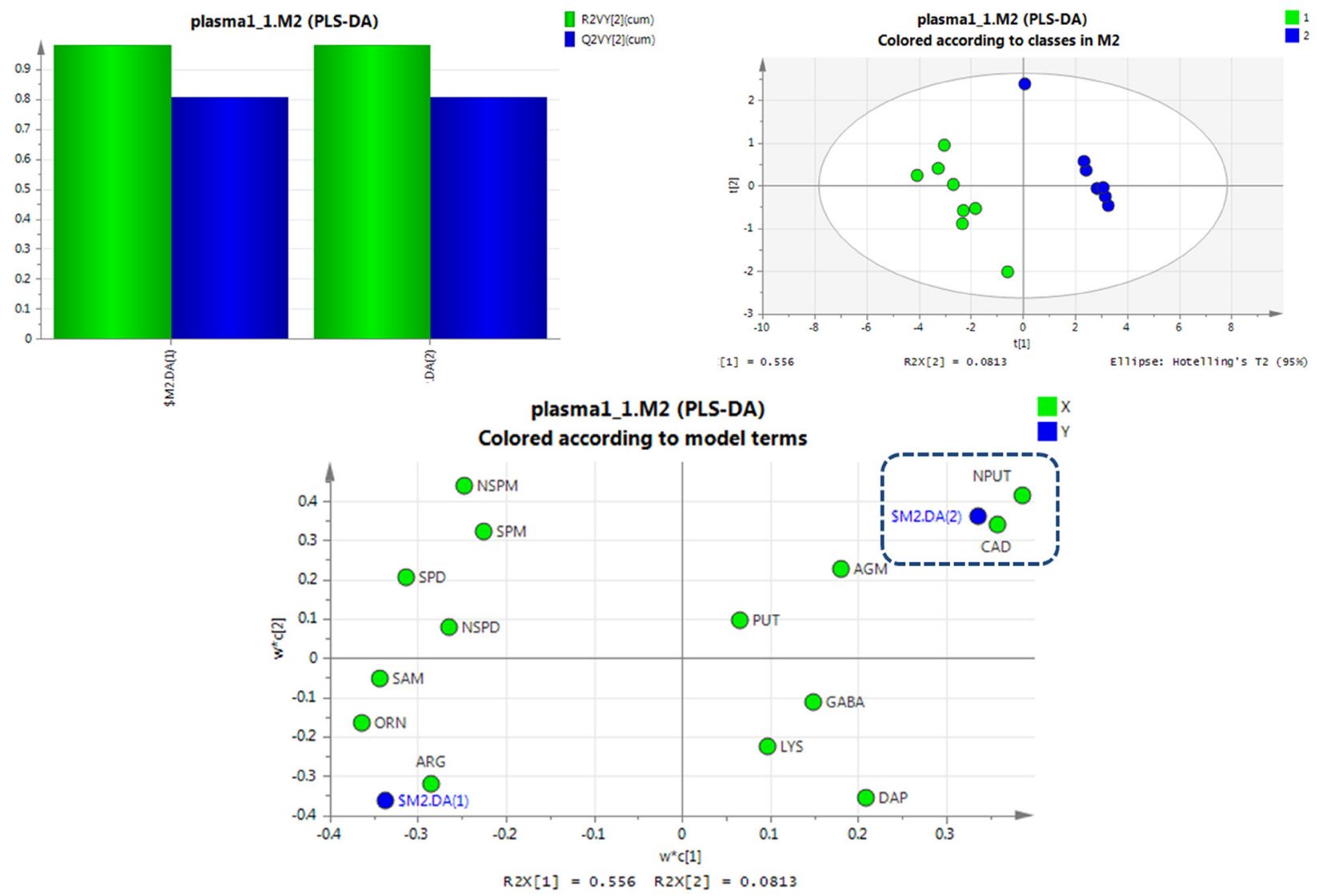

Figure 3: PLS-DA analysis of healthy rats and SCCL rats suffered suffered carcinogenesis for 28 days using polyamines as indicators. (DAP: 1,3-diaminopropane, PUT: putrescine, CAD: cadaverine, SPM: spermine, SPD: spermidine, NPUT: N-acetylputrescine, NSPM: N-acetylspermine, NSPD: N-acetylspermidine, GABA: $\gamma$-aminobutyric acid, AGM: agmatine, ARG: L-arginine, LYS: lysine, ORN: L-ornithine, SAM: S-adenosyl-L-methionine). 
from S-adenosy-L-methionine by S-adenosylmethionine decarboxylase, is involved in the formation of spermidine and spermine. The increased level of 1,3-diaminopropane in the development of SCCL and its reduced level in the treatment of SCCL can be interpreted as showing that the interactions of spermidine and spermine with DNA analogues can be changed during the progression of cancer. For the same reason, cadaverine, derived from lysine, could serve as a cancer indicator during the treatment stage of SCCL.

\section{MATERIALS AND METHODS}

\section{Materials and treatments}

The reference standards of 1,3-diaminopropane, putrescine, cadaverine hydrochloride, spermidine hydrochloride, spermine, agmatine sulfate salt, $\mathrm{N}$-acetylputrescine hydrochloride, N-acetylspermine trihydrochloride, $\mathrm{N}$-acetylspermidine dihydrochloride, L-ornithine hydrochloride, lysine, L-arginine,
S-adenosyl-L-methionine, $\gamma$-aminobutyric acid and 1 , 6-diaminohexane (used as an internal standard) were all obtained from Sigma-Aldrich (St. Louis, MO). HPLC grade methanol was purchased from Fisher Chemicals (Fair Lawn, NJ). Heptafluorobutyric acid (HFBA) was obtained from Sigma-Aldrich (St. Louis, MO) and all the other reagents were of analytic grade. Redistilled and deionized water was used throughout the study.

3-Methylcholanthrene and diethylnitrosamine were obtained from Sigma-Aldrich (St. Louis, MO) and 5-fluorouracilat were purchased from Shanghai Xudong Haipu Pharmaceutical CO., LTD. (Shanghai, China). Aidi injections are an effective Chinese herbal preparations with anti-cancer activity used for the treatment of liver, lung and colorectal cancer, and they have been used clinically for many years $[24,25]$. The Aidi injections were prepared according to the Ministerial Standards of Chinese Medicine. They contain Mylabris (the dried polypide of Mylabris phalerata Pall or Mylabris cichorii Linnaeus), Ginseng Radix Et Rhizoma (the dried root and rhizome of Panax ginseng C.A.Mey.), Astragali Radix (the dried root of Astragalus
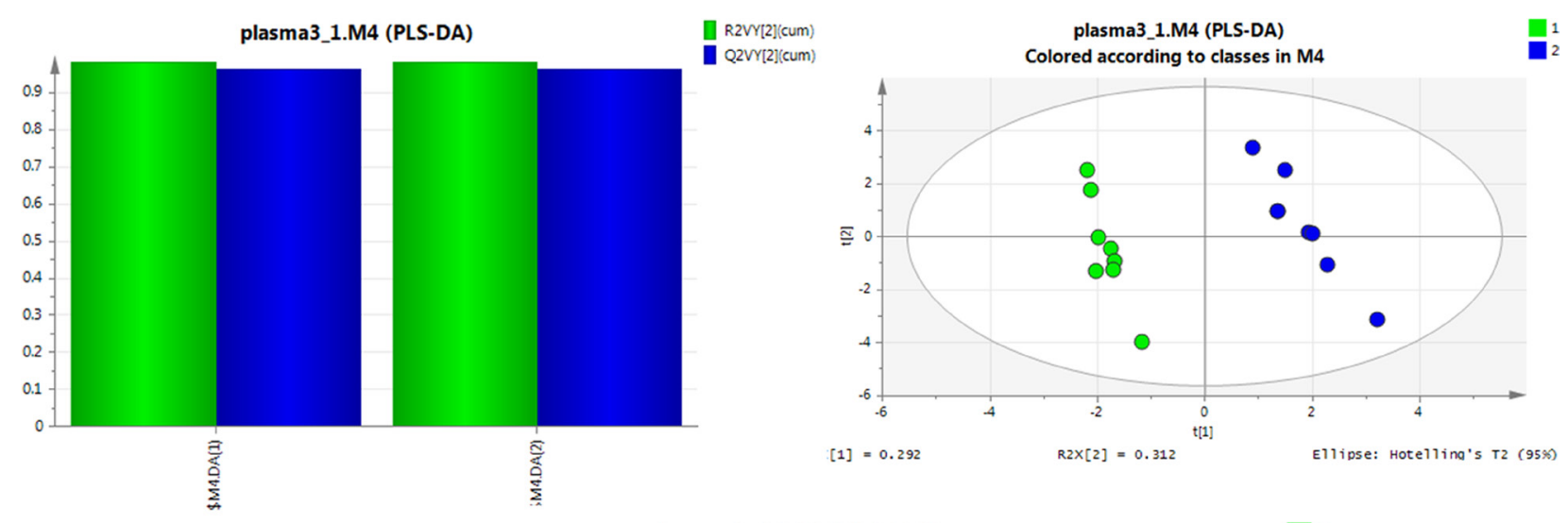

plasma3_1.M4 (PLS-DA)

$[1]=0.292$

$R 2 \times[2]=0.312$

Ellipse: Hotellina's T2 (95\%)

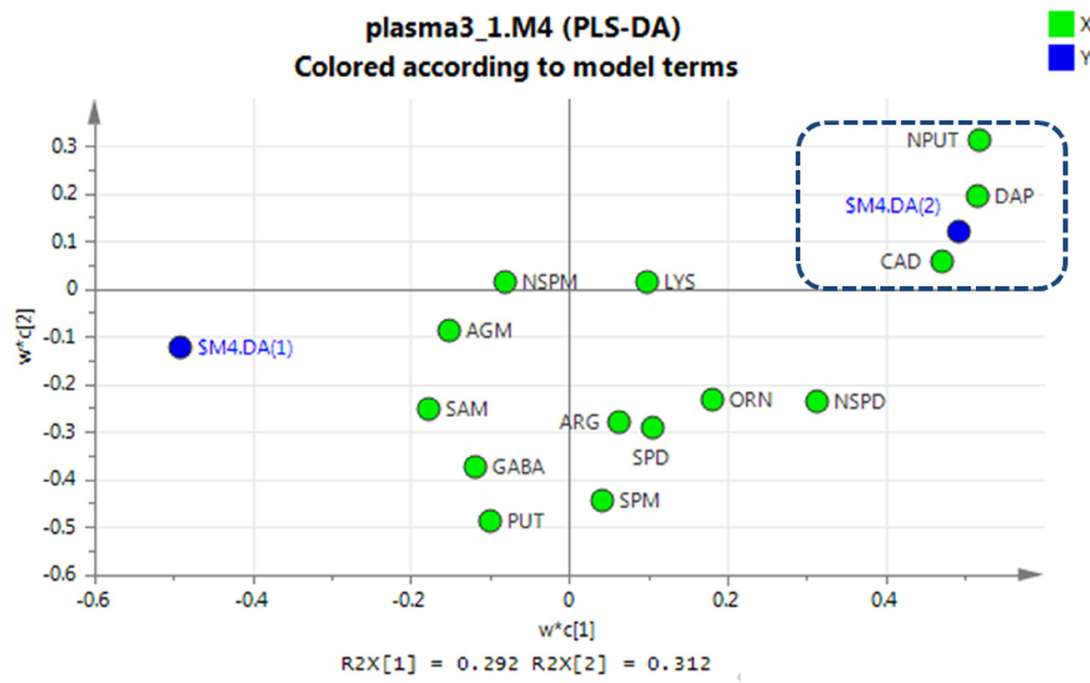

Figure 4: PLS-DA analysis of healthy rats and SCCL rats suffered suffered carcinogenesis for 70 days using polyamines as indicators. (DAP: 1,3-diaminopropane, PUT: putrescine, CAD: cadaverine, SPM: spermine, SPD: spermidine, NPUT: N-acetylputrescine, NSPM: N-acetylspermine, NSPD: N-acetylspermidine, GABA: $\gamma$-aminobutyric acid, AGM: agmatine, ARG: L-arginine, LYS: lysine, ORN: L-ornithine, SAM: S-adenosyl-L-methionine). 
membranaceus (Fisch.) Bge.var.mongholicus (Bge.) Hsiao, and Acanthopanacis Senticosi Radix Et Rhizoma Seu Caulis (the dried root and rhizome or of Acanthopanax senticosus (Rupr.et Maxim.) Harms). All the treatments used in our experiments were prepared immediately prior to use.

The activities of CYFRA21-1 and SCCAg were determined using ELISA (Enzyme Linked Immunosorbent Assay) assay kits according to the manfacturer's instructions (Supplementary Table 4 listed the detailed results of ELISA assay).

\section{Experimental animals and sample collection}

Ninety-six male and female Wistar rats (200-220 g) were obtained from the Experimental Animal Center of Shenyang Pharmaceutical University and fed standard rodent chow and given unlimited access to water in an air-conditioned animal center at a temperature of $22 \pm 2^{\circ} \mathrm{C}$ and a relative humidity of $50 \pm 10 \%$, with a natural light-dark cycle during the experiment period. The animal studies were carried out according to the
Guidelines of Animal Experimentation of Shenyang Pharmaceutical University, and the protocol was approved by the Animal Ethics Committee of the institution.

The study was performed after the rats allowed to acclimatize for one week. The rats were first divided into two groups, SCCL model rats $(n=72)$ and normal rats $(n=24)$. After basal anesthesia produced by intraperitoneal injection of $10 \%$ chloral hydrate, the SCCL model rats had cancer induced by intratracheal instillation of 3-methylcholanthrene (MCA, $10 \mathrm{mg} / 0.1 \mathrm{ml}$ ), while diethylnitrosamine (DEN, $0.01 \mathrm{mg} / 0.1 \mathrm{ml}$ ) was also given as the oncogenic agent [26-27]. Normal rats received the same volume of vehicle (iodipin) without MCA and DEN at the same time. During the period of carcinogenesis, plasma and lung samples were collected from the SCCL model rats $(n=8)$ and normal rats $(n=8)$ on the 28th day. Then, on the 42th day, the SCCL model rats $(n=64)$ were divided into four groups, an SCCL group $(n=16)$, a 5 -fluorouracil treated group (given 5-fluorouracil injections of $20 \mathrm{mg} / \mathrm{kg}$ once a week, $n=16$ ), an Aidi injection group (given Aidi injections of $4 \mathrm{~mL} / \mathrm{kg}$ three times a week,
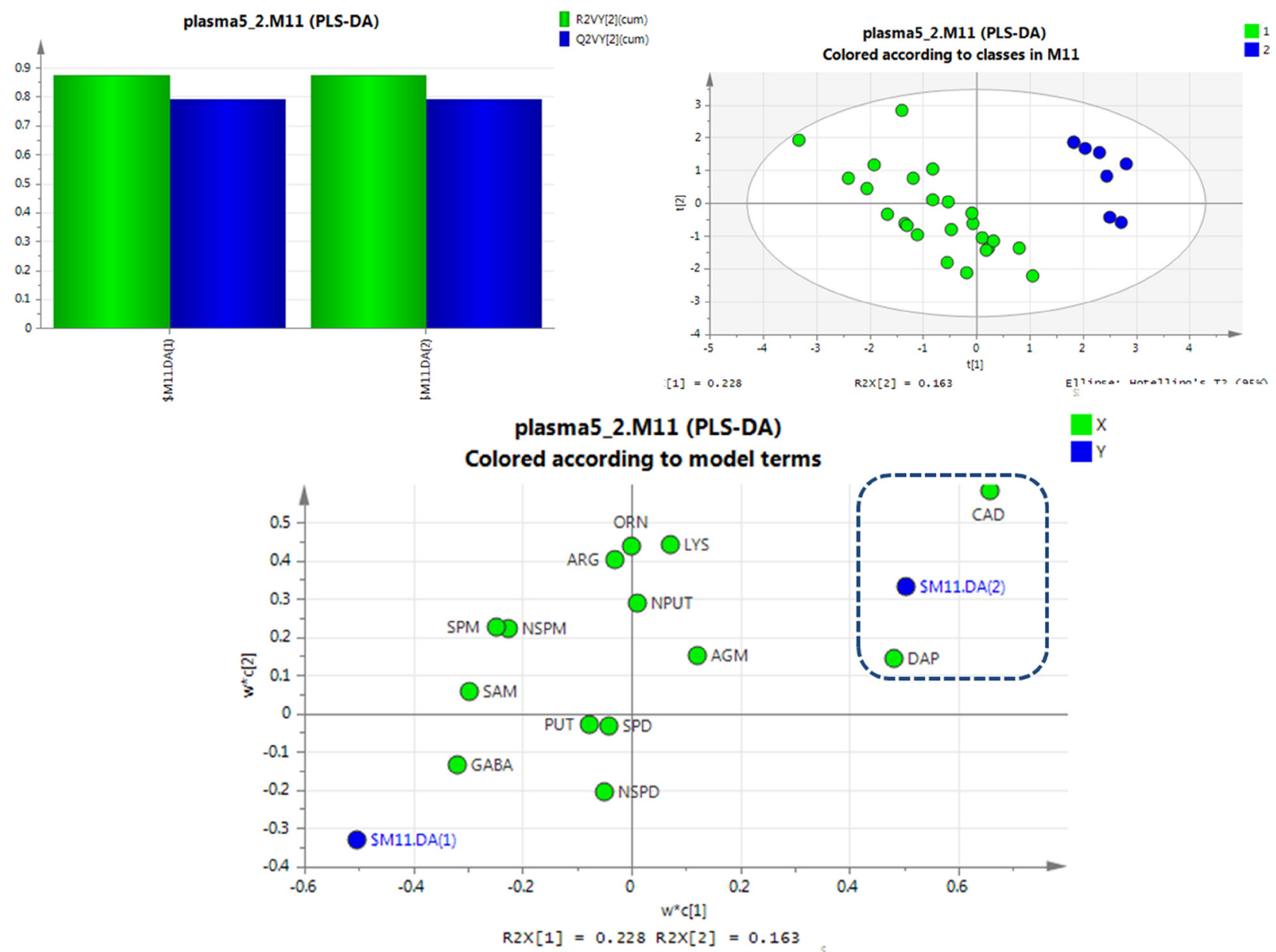

Figure 5: PLS-DA analysis of SCCL rats and treated rats given different treatments for 56 days using polyamines as indicators. (DAP: 1,3-diaminopropane, PUT: putrescine, CAD: cadaverine, SPM: spermine, SPD: spermidine, NPUT: N-acetylputrescine, NSPM: N-acetylspermine, NSPD: N-acetylspermidine, GABA: $\gamma$-aminobutyric acid, AGM: agmatine, ARG: L-arginine, LYS: lysine, ORN: L-ornithine, SAM: S-adenosyl-L-methionine). 
$n=16$ ), and a combined medication group (given Aidi injections three times a week and 5-fluorouracil injections once a week, $n=16$ ). On the 70th and 98th day, twentyfour hours after the last administration, plasma and lung samples were collected from each group ( $n=8$ for each group) and immediately frozen at $-80^{\circ} \mathrm{C}$ until analysis. Histopathological examination on lung samples was used to confirm the success of the SCCL model and to examine the therapeutic effects of the medicines. Each group contained aqual numbers of male and female rats throughout the experiment.

\section{Polyamine detection and analysis}

For determination of the polyamine metabolome, we used a simple and sensitive UHPLC-MS/MS method described in detail in our previous paper [23]. Briefly, chromatographic separation was achieved with gradient elution using a mobile phase composed of $0.05 \%$ heptafluorobutyric acid (HFBA) in water (A) and $0.05 \%$ HFBA in methanol (B). MS detection was carried out using a QTRAPTM 4000 MS/MS system from Applied AB Sciex equipped with a Turbo Ion Spray source
(Foster City, CA, USA). The detection of the analytes was in multiple reaction monitoring mode (MRM) using electrospray positive ionization $\left(\mathrm{ESI}^{+}\right)$. Each $250 \mu \mathrm{L}$ plasma sample was deproteinization by the addition of $250 \mu \mathrm{L}$ methanol (containing $0.1 \%$ acetic acid), vortex mixed for $5 \mathrm{~min}$ and then centrifuged for $3 \mathrm{~min}$ at $15,000 \mathrm{rpm}$ and $4^{\circ} \mathrm{C}$. The supernatant was transferred to another Eppendorf microtube and then evaporated to dryness at $30^{\circ} \mathrm{C}$ under a stream of air. The residue was dissolved in $50 \mu \mathrm{L}$ methanol (containing $0.05 \%$ HFBA) -water (containing $0.05 \%$ HFBA) $(20: 80, v / v)$ and $5 \mu \mathrm{L}$ of the supernatant was injected for analysis. Lung samples were first weighed on an electronic analytical balance from METTLER TOLEDO (Greifensee, Switzerland), and then homogenized in a 10-fold volume of methanol-water $(20: 80, v / v)$, vortex mixed for $5 \mathrm{~min}$ and centrifuged for 3 min at $15,000 \mathrm{rpm}$ and $4^{\circ} \mathrm{C}$, and $5 \mu \mathrm{L}$ of supernatant was injected for analysis.

\section{Statistical testing}

Polyamine concentrations were obtained from calibration curves and expressed as mean \pm SD. The
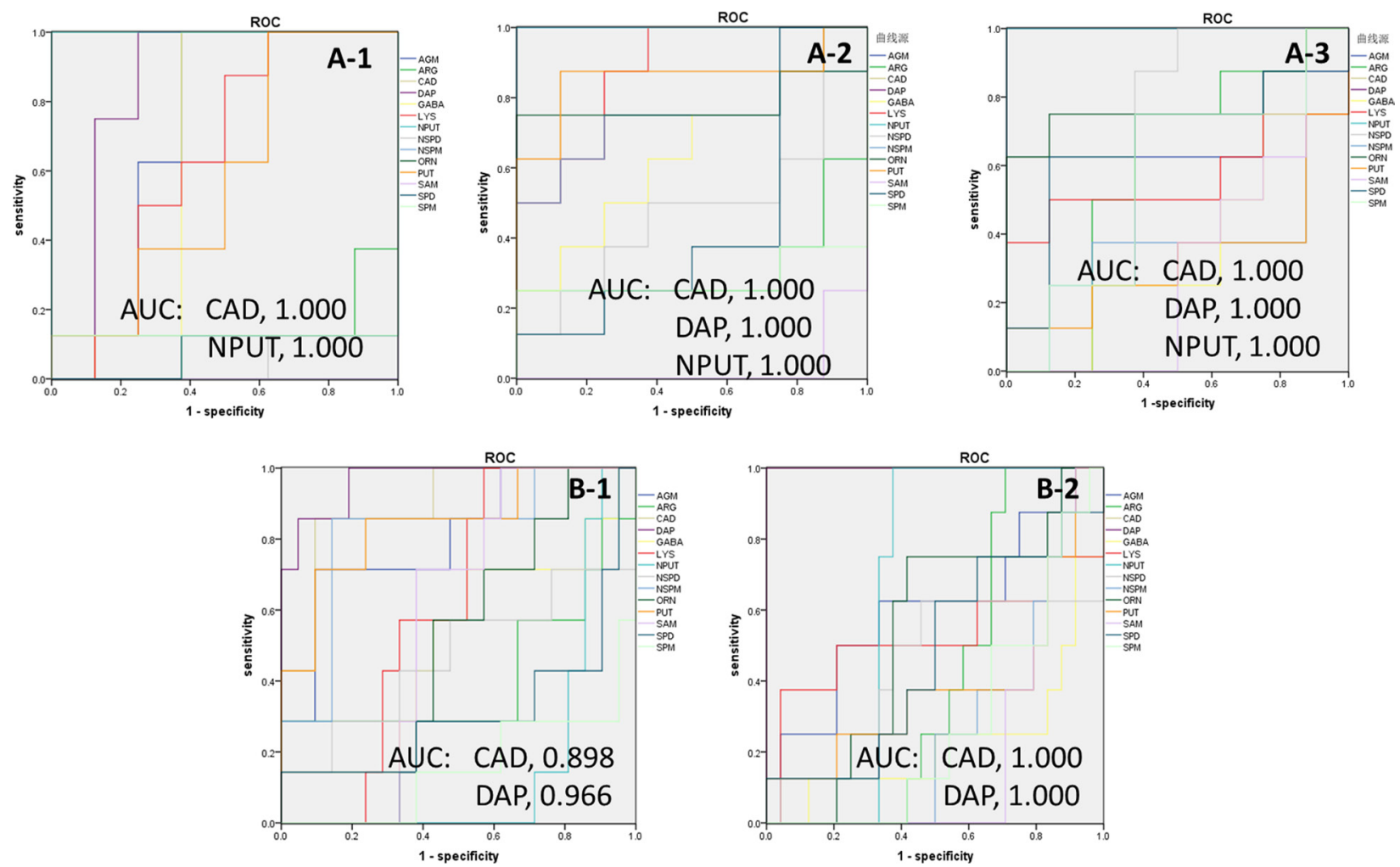

Figure 6: Univariate receiver operating characteristic curve (ROC) analyses for biomarker identification. Using a bootstrapping approach to compute the $95 \%$ confidence interval (CI) for a single cut-off or for the complete ROC curve. The AUC value of the potential biomarkers for SCCL and treatment are plotted. A-1: SCCL model rats after carcinogenesis for 28 days; A-2: SCCL model rats after carcinogenesis for 70 days; A-3: SCCL model rats after carcinogenesis for 98 days; B-1: SCCL model rats after therapeutic treatment by three kinds of drug for 28 days; B-2: SCCL model rats after therapeutic treatment by three kinds of drug for 56 days; (DAP: 1,3-diaminopropane, PUT: putrescine, CAD: cadaverine, SPM: spermine, SPD: spermidine, NPUT: N-acetylputrescine, NSPM: N-acetylspermine, NSPD: N-acetylspermidine, GABA: $\gamma$-aminobutyric acid, AGM: agmatine, ARG: L-arginine, LYS: lysine, ORN: L-ornithine, SAM: S-adenosyl-L-methionine). (Supplementary Tables 1 and 2 listed the detailed results of ROC analysis). 
results were presented as mean $\pm \mathrm{SD}$ and analyzed statistically with Student's $t$-test and the nonparametric Mann-Whitney test using SPSS 19.0 software for Windows (SPSS Inc., Chicago, IL). The threshold of significance was set at $P<0.05$. PLS-DA and ROC analysis was also carried out using SIMAC-P (Umetrics AB, Umea, Sweden) and SPSS 19.0, respectively.

\section{CONCLUSIONS}

A plasma polyamine metabolic profile analysis was performed using UHPLC-MS/MS detection following intratracheal instillation of 3-methylcholanthrene in rats during the process of SCCL development and treatment, and 14 polyamine metabolome were detected. Combinating PLS-DA and ROC analysis, it had been shown that $\mathrm{N}$-acetylputrescine and 1,3-diaminopropane may be used as biomarkers to diagnose lung cancer at an early stage, as they were significantly increased in SCCL rats compared with healthy rats. Also, during treatment with the three therapeutic options, the levels of cadaverine and 1,3-diaminopropane decreased with anti-cancer therapy until they become equal to the levels in the control rats, and could be used as biomarkers for evaluating the therapeutic effect of anti-cancer drugs on SCCL. These results may explain the metabolic disturbance in SCCL progression and their recovery by the anti-cancer drugs. Furthermore, the present polyamine profiling combined with quantitative targeted metabolomics methodology will be useful for early cancer screening and anti-cancer drug monitoring.

\section{ACKNOWLEDGMENTS AND FUNDING}

This study was financially supported by the National Natural Science Funds of China (Grant No. 81603277/ H2803 and Grant No. 81102784/H2803).

\section{CONFLICTS OF INTEREST}

The authors declare no conflicts of interest.

\section{REFERENCES}

1. PDQ Adult Treatment Editorial Board. Non-Small Cell Lung Cancer Treatment $\left(\mathrm{PDQ}^{\circledR}\right)$ : Health Professional Version. PDQ Cancer Information Summaries [Internet]. Bethesda (MD): National Cancer Institute (US). 2002-2017.

2. Jemal A, Ward EM, Johnson CJ, Cronin KA, Ma J, Ryerson B, Mariotto A, Lake AJ, Wilson R, Sherman RL, Anderson RN, Henley SJ, Kohler BA, et al. Annual Report to the Nation on the Status of Cancer, 1975-2014, Featuring Survival. J Natl Cancer Inst. 2017; 109. https:// doi.org/10.1093/jnci/djx030.
3. Corrales L, Nogueira A, Passiglia F, Listi A, Caglevic C, Giallombardo M, Raez L, Santos E, Rolfo C. SecondLine Treatment of Non-Small Cell Lung Cancer: Clinical, Pathological, and Molecular Aspects of Nintedanib. Front Med (Lausanne). 2017; 4:13. https://doi.org/10.3389/ fmed.2017.00013.

4. Zhao T, Jin Y, Mao G, Wei Y, Wu G, Ye X, Zhou Y, Yuan G, Gao L, Hong Y, Chen Y, Hong C, Zhou H, et al. CYFRA 21-1 is an early predictor of chemotherapeutic effectiveness in advanced nonsmall cell lung cancer: An observational study. Medicine (Baltimore). 2016; 95:e5748.

5. Williams M, Swampillai A, Osborne M, Mawdsley S, Hughes R, Harrison M, Harvey R, Glynne-Jones R; Mount Vernon Colorectal Cancer Network. Squamous cell carcinoma antigen: a potentially useful prognostic marker in squamous cell carcinoma of the anal canal and margin. Cancer. 2013; 119:2391-2398.

6. Touloupidis S, Zisimopoulos A, Giannakopoulos S, Papatsoris AG, Kalaitzis C, Thanos A. Clinical usage of the squamous cell carcinoma antigen in patients with penile cancer. Int J Urol. 2007; 14:174-76.

7. Holdenrieder S. Scand, Biomarkers along the continuum of care in lung cancer. J Clin Lab Invest Suppl. 2016; 245:S40-45.

8. Di Gioia D, Blankenburg I, Nagel D, Heinemann V, Stieber P. Tumor markers in the early detection of tumor recurrence in breast cancer patients: CA 125, CYFRA 21-1, HER2 shed antigen, LDH and CRP in combination with CEA and CA 15-3. Clin Chim Acta. 2016; 461:1-7.

9. Calvayrac O, Pradines A, Pons E, Mazières J, Guibert N. Molecular biomarkers for lung adenocarcinoma. Eur Respir J. 2017; 49. https://doi.org/10.1183/13993003.01734-2016.

10. Hu J, Liu YL, Piao SL, Yang DD, Yang YM, Cai L. Expression patterns of USP22 and potential targets BMI-1, PTEN, p-AKT in non-small-cell lung cancer. Lung Cancer. 2012; 77:593-599.

11. Brennan L. Session 2: Personalised nutrition. Metabolomic applications in nutritional research. The Proceedings of the Nutrition Society, 2008; 67:404-408.

12. Mamas M, Dunn WB, Neyses L, Goodacre R. The role of metabolites and metabolomics in clinically applicable biomarkers of disease. Archives of Toxicology. 2011; 85: 5-17.

13. Barnes S, Benton HP, Casazza K, Cooper SJ, Cui X, Du X, Engler J, Kabarowski JH, Li S, Pathmasiri W, Prasain JK, Renfrow MB, Tiwari HK. Training in metabolomics research. II. Processing and statistical analysis of metabolomics data, metabolite identification, pathway analysis, applications of metabolomics and its future. J Mass Spectrom. 2016; 51:535-548.

14. Checkley W, Deza MP, Klawitter J, Romero KM, Klawitter J, Pollard SL, Wise RA, Christians U, Hansel NN. Identifying biomarkers for asthma diagnosis using targeted metabolomics approaches. Respir Med. 2016; 121:59-66. 
15. Stevens AP, Dettmer K, Kirovski G, Samejima K, Hellerbrand C, Bosserhoff AK, Oefnera PJ. Quantification of intermediates of the methionine and polyamine metabolismby liquid chromatography-tandem mass spectrometry in cultured tumor cellsand liver biopsies. Journal of Chromatography A. 2010; 1217:3282-3288.

16. Huang Y, Pledgie A, Casero RA Jr, Davidson NE. Molecular mechanisms of polyamine analogs in cancer cells. Anticancer Drugs. 2005; 16:229-241.

17. Moinard C, Cynobera L, de Bandt JP. Polyamines: metabolism and implicationsin human diseases. Clinical Nutrition. 2005; 24:184-197.

18. Paik MJ, Kuon D, Cho J, Kim KR. Altered urinary polyamine patterns of cancer patients under acupuncture therapy. Amino Acids. 2009; 37:407-413.

19. Eugene W. Gerner, Cancer Chemoprevention Locks onto a New Polyamine Metabolic Target. Cancer Prev Res. 2010; $3: 125-127$.

20. Agostinelli E, Marques MPM, Calheiros R, Gil FPSC, Tempera G, Viceconte N, Battaglia V, Grancara S, Toninello A. Polyamines: fundamental characters in chemistry and biology. Amino Acids. 2010; 38:393-403.

21. Liu R, Jia Y, Cheng W, Ling J, Liu L, Bi K, Li Q. Determination of polyamines in human urine by precolumn derivatization with benzoyl chloride and high-performance liquid chromatography coupled with Q-time-of-flight mass spectrometry. Talanta. 2011; 83:751-756.

22. Liu R, Bi K, Jia Y, Wang Q, Yin R, Li Q. Determination of polyamines in human plasma by high-performance liquid chromatography coupled with Q-TOF mass spectrometry. J Mass Spectrom. 2012; 47:1341-1346.
23. Liu R, Li Q, Ma R, Lin X, Xu H, Bi K. Determination of polyamine metabolome in plasma and urine by ultrahigh performance liquid chromatography-tandem mass spectrometry method: Application to identify potential markers for human hepatic cancer. Analytica Chimica Acta. 2013; 791:36-45.

24. Xu HX, Huang XE, Li Y, Li CG, Tang JH. A clinical study on safety andefficacy of Aidi injection combined with chemotherapy, Asian Pac. J Cancer Prev. 2011; 12: 2233-2236.

25. Sun XF, Pei YT, Yin QW, Wu MS, Yang GT. Application of Aidi injection inthe bronchial artery infused neo-adjuvant chemotherapy for stage III Anon-small cell lung cancer before surgical operation. Chin J Integr Med. 2010; 16:537-541.

26. Liu WB, Cui ZH, Ao L, Zhou ZY, Zhou YH, Yuan XY, Xiang YL, Liu JY, Cao J. Aberrant methylation accounts for cell adhesion-related gene silencing during 3-methylcholanthrene and diethylnitrosamine induced multistep rat lung carcinogenesis associated with overexpression of DNA methyltransferases 1 and $3 \mathrm{a}$. Toxicol Appl Pharmacol. 2011; 251:70-78.

27. Liu WB, Ao L, Zhou ZY, Cui ZH, Zhou YH, Yuan XY, Xiang YL, Cao J, Liu JY. CpG island hypermethylation of multiple tumor suppressor genes associated with loss of their protein expression during rat lung carcinogenesis induced by 3-methylcholanthrene and diethylnitrosamine. Biochem Biophys Res Commun. 2010; 402:507-514. 